\title{
Homogenization of single-walled carbon nanotubes
}

\author{
Tanguy Messager, Patrice Cartraud
}

Institut de Recherche en Génie civil et Mécanique (GeM), UMR CNRS 6183

Ecole Centrale de Nantes, BP 92101, 44321 Nantes cédex3, France

tanguy.messager@univ-nantes.fr, patrice.cartraud@ec-nantes.fr

\begin{abstract}
This work deals with the computation of the overall axial elastic behavior of single-walled carbon nanotubes (SWCNTs). The SWCNTs are modeled as space-frame structures, using beam elements to represent atomic bonds [1].

The application of homogenization theory [2] enables to derive rigorously the macroscopic anisotropic beam behavior of the SWCNT, from the solution of three-dimensional basic cell problems. Moreover, taking benefit of the two helical symmetries [3] of the microstructure, the basic cell can be reduced to only one half of an hexagon, as depicted in Fig.1 for a zigzag SWCNT. Therefore, the overall stiffness coefficients can be computed efficiently using very concise FE models (including only 3 beam elements): the helical symmetry properties of the displacement field lead to a set of linear relationships expressed in local cylindrical axes between the opposite nodes $m$ and $n$ (see Fig.1) then acting as boundary conditions [3].

The accuracy of this approach has been assessed with respect to reference solutions of the literature [1] and also from comparison with results given by large FE model (left part of Fig.1). This method has been applied for the computation of zigzag and armchair SWCNTs: as shown in Fig.2, the developed procedure allows to study the scale effects.
\end{abstract}

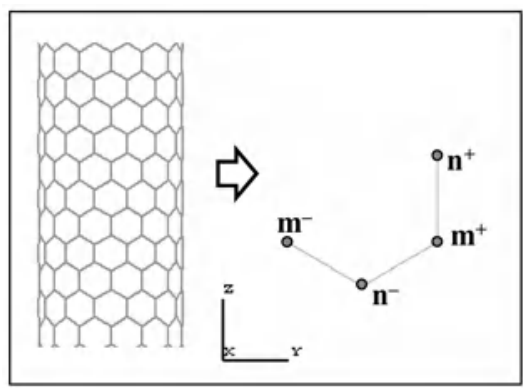

Fig.1: Overall structure and microscopic FE model

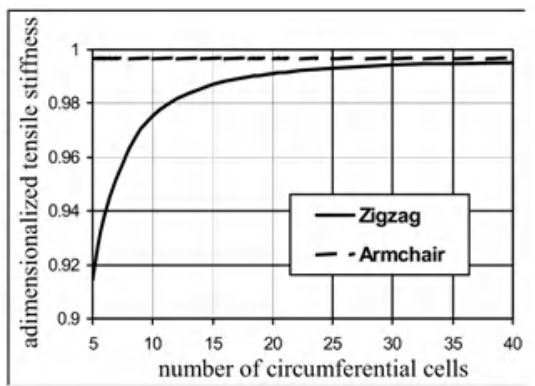

Fig.2: tensile stiffness evolution

\section{References}

[1] K.I. Tserpes, P. Papanikos, Finite element modeling of single-walled carbon nanotubes. Composites Part B, 36, 468-477, 2005.

[2] P. Cartraud, T. Messager, Computational homogenization of periodic beam-like structures. Int. J. of Solids and Structures, in press, 2005.

[3] T. Messager, P. Cartraud, Homogenization of helical beam-like structures. Finite Elements in Analysis and Design, submitted, 2005. 